\title{
Left coronary artery fistula to right ventricle complicated heart failure in a patient on hemodialysis
}

\author{
Egidio Imbalzano • Giuseppe Dattilo • \\ Mirko Scarpelli • Alberto Lo Gullo • \\ Antonino Saitta
}

Received: 1 August 2013/Accepted: 21 August 2013/Published online: 30 August 2013

(c) SIMI 2013

Coronary artery fistulas (CAF) are rare, and are most often diagnosed by echocardiography or by coronary angiography. The incidence of this disease is very low, with a more frequent occurrence of fistulas originating in the right coronary artery. There is a higher incidence of CAF to right heart chambers, with CAF to the left ventricle (LV) being rare. Treatment can be surgical or percutaneous [1]. This report describes a case of CAF to the right ventricle $(\mathrm{RV})$ resulting in severe pulmonary hypertension, in a patient with end-stage renal disease (ESRD) on hemodialysis and rheumatoid arthritis [2]. The patient had a history of hypertension for over 30 years [3]. Computed tomographic pulmonary angiography ruled out pulmonary embolism, but it suggested a coronary fistula to the RV cavity.

E. Imbalzano $(\varangle) \cdot$ G. Dattilo · M. Scarpelli · A. Lo Gullo ·

A. Saitta

Department of Internal Medicine, University of Messina,

Messina, Italy

e-mail: eimbalzano@unime.it
A 61-year-old woman was referred for cardiac evaluation for chest pain and worsening dyspnea. The patient was on maintenance hemodialysis for the prior 2 years owing to ESRD. Clinical examination revealed tachycardia, crackles at both bases with no wheezes, rhonchi, or other adventitious sounds, a blood pressure of $160 / 70 \mathrm{mmHg}$. The patient had a radio-cephalic fistula constructed at the left wrist between the radial artery and the cephalic vein, using end-to-end anastomosis. An electrocardiogram showed evidence of a right bundle branch block, and signs of ventricular overload. Cross-sectional echocardiography with Doppler interrogation revealed a left ventricular ejection fraction of $55 \%$, and there was evidence of diastolic dysfunction, without important calcification or regurgitation of the valves.

The right atrium and ventricle were dilatated (Fig. 1a) with an abnormal flow jet pointed to the RV (Fig. 1b), and evidence of severe systolic pulmonary hypertension (PHT) (Fig. 1c) estimated to be $80 \mathrm{mmHg}$ on continuous wave Doppler. There was moderate tricuspid regurgitation with RV function moderately depressed (tricuspid anular plane systolic excursion: $13 \mathrm{~mm}$ ) [4]. To obtain precise information on the cause of the PHT, a 64-slice multidetector computed tomography (MDCT) was performed. MDCT confirmed right heart dilatation and revealed a CAF connecting the anterior descending artery to the RV cavity without any evidence of pulmonary thromboembolism (Fig. 1d). A coronary angiography showed an enlarged and tortuous aneurysm with a CAF (Fig. 1e) that protruded into the RV, with immediate closure percutaneous with a detachable helical coil (Fig. 1f). We doubt that it was a secondary form, manifested as a result of treatment of hemodialysis causing right heart failure. 
Fig. 1 a Right atrium and ventricle were dilated. b Abnormal flow jet (white arrow) from through the fistula to the RV. c Severe systolic pulmonary hypertension. d Multidetector computed tomography (MDCT) confirmed right heart dilatation and revealed suspicion of fistula connecting the anterior descending artery to RV cavity. e Coronary angiography showing enlarged and tortuous aneurysm with a CAF (white arrow) which protrude into the $\mathrm{RV}$. f CAF has been occluded with a detachable helical coil, wedged into the proximal neck of the CAF (white arrow)
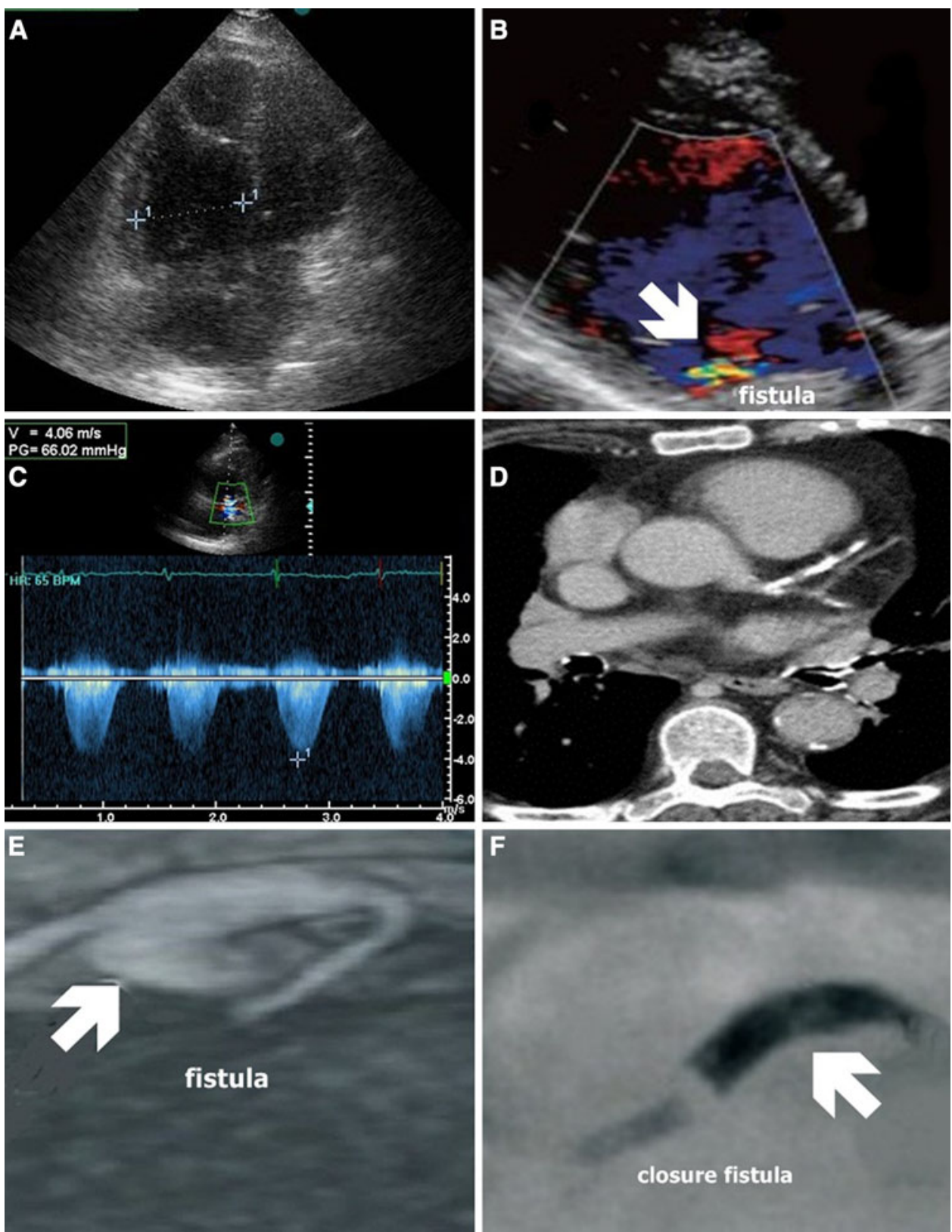

Conflict of interest None.

\section{References}

1. Katona A, Ungi I, Forster T, Katona M, Nemes A (2013) A single coronary artery with a right ventricular fistula : unique therapy with covered-stent implantation. Herz [Epub ahead of print]

2. Tiritilli A, Iaria P, Viard P, Sayah S, Benali T, Detienne JP, Martis S, Tchatchum F, Aouate P (2013) Coronary artery fistulas, a current problem: clinical and therapeutic considerations. Ann Cardiol Angeiol (Paris) [Epub ahead of print]

3. Sardo MA, Mandraffino G, Campo S, Saitta C, Bitto A, Alibrandi A, Riggio S, Imbalzano E, Saitta A (2009) Biglycan expression in hypertensive subjects with normal or increased carotid intimamedia wall thickness. Clin Chim Acta 406(1-2):89-93

4. Zhang Y, Song YH, Liu ZG, Wang W (2012) Large right coronary artery to left ventricular fistula. J Card Surg 27(3):311-313 